\title{
Diagnostic value of serum homocysteine and blood lipid level in different types of coronary atherosclerotic cardiopathy.
}

\author{
Huiling Zhang ${ }^{1,2}$, Jun Yang ${ }^{1,3 *}$ \\ ${ }^{1}$ Qingdao University, Qingdao, Shandong, PR China \\ ${ }^{2}$ Department of Cardiology, Affiliated Hospital of Jining Medical University, Shandong, PR China \\ ${ }^{3}$ Department of Cardiology, Yantai City Yuhuangding Hospital, Shandong, PR China
}

\begin{abstract}
Objective: The incidence of coronary heart disease is rapidly rising in China. The relationship of serum homocysteine (Hcy) and blood lipid levels with Coronary Heart Disease (CHD) is still controversy. This study analysed the relationship of serum Hcy and blood lipid level in different types of CHD.

Patients and methods: A total of 100 cases of CHD patients in different types and healthy volunteers were selected. Serum Hcy, Triglyceride (TG), High Density Lipoprotein (HDL), and Low Density Lipoprotein (LDL) were measured. The levels of serum Hcy and blood lipid levels were compared in CHD patients with different clinical types, vascular lesions, and coronary stenosis by chi-square test. The sensitivity and specificity of serum Hcy and blood lipid levels in CHD were evaluated. The relationship between serum Hcy and blood lipid levels were analysed by linear regression analysis.

Results: Hcy, TP, TG, and LDL were significantly higher, while HDL level was obviously lower in experimental group compared with control $(\mathbf{P}<0.05)$. The levels of serum Hcy, TP, TG, and LDL gradually increased, whereas HDL level gradually declined among SAP, UAP, and AMI patients, in single vessel disease group, double vessels disease group, and multivessel disease group, and in patients with mild, moderate, severe stenosis, and entirely occlusion $(\mathbf{P}<0.05)$. Serum HDL exhibited high sensitivity, while serum Hcy presented high specificity.

Conclusion: Hcy and blood lipid levels increased in CHD. Hcy and blood lipid level exhibited no correlation with each other. Their combination detection is of diagnostic value to CHD.
\end{abstract}

Keywords: Homocysteine, Blood lipid level, Coronary heart disease D.

Accepted on November 14, 2017

\section{Introduction}

Coronary Atherosclerotic Heart Disease (CHD) is a type of myocardial dysfunction disease mostly due to coronary artery stenosis, resulting in coronary insufficiency [1]. In recent years, the incidence of CHD increased year by year with living standard improving. According to the report published by World Health Organization in 2011, the death due to CHD in China had ranked second around the world with younger trend [2]. It was found that in addition to age, gender, genetic factors, smoking, hypertension, hyperlipidemia, and diabetes, coagulation fibrillation imbalance, inflammation, and infectious diseases were also important factors on the development of CHD. Therefore, in-depth study in the relevant areas of CHD should be focused. Timely and accurate diagnosis of CHD has an important clinical value [3], which is the basic method to reduce the injury of CHD.

The formation of Atherosclerosis (AS) is affected by many factors. Homocysteine (Hcy) is a methionine metabolite belonging to sulfur-containing amino acids. It was suggested that Hcy level may be related to AS [4]. Recently, it was reported that total Hcy elevation is an independent risk factor for AS and a new risk factor for CHD. Hcy causes lipid accumulation and other aspects in damaging the vascular wall and affecting coagulation factors [5]. Meanwhile, numerous clinical trials confirmed that blood lipid levels, including TP, HDL, and LDL, played crucial roles in the occurrence and development of AS played an important role [6]. In this study, we selected CHD patients admitted to our hospital and tested the expressions of Hcy and lipid indicators to analyse the relationship of serum Hcy and blood lipid level in different types of CHD.

\section{Materials and Methods}

\section{General information}

A total of 100 CHD patients in Affiliated Hospital of Jining Medical University from January 2016 to January 2017 diagnosed by coronary angiography were selected. There were 
56 males and 44 females with mean age at $48.9 \pm 3.4(35-70 \mathrm{y}$ old). The subjects were divided into Stable Angina Pectoris group (SAP) (35 cases), Unstable Angina Pectoris group (UAP) (35 cases), and Acute Myocardial Infarction group (AMI) (30 cases) according to different clinical types. The subjects were further divided into single-vessel disease group (31 cases), double-vessel disease group (37 cases), and multivessel disease group (32 cases) according to the result of coronary arteriography. Another 100 healthy volunteers were enrolled as control group, including 64 males and 36 females with an average age of $50.1 \pm 1.6(40-70 \mathrm{y})$. There were no significant differences in gender, age, and other general data between the two groups $(\mathrm{P}>0.05)$. The study was approved by the medical ethics committee in our hospital and all the subjects had provided informed consent.

\section{Exclusion criteria}

Acute myocardial infarction, unstable angina, or cerebrovascular disease within 3 months; III or IV grade heart failure according to the New York Heart Disease Association (NYHA) grade; other heart disease; acute or chronic infectious disease; malignancy; autoimmune disease; diabetes; kidney disease; lactation and pregnancy patients; and liver dysfunction [7].

\section{Experimental methods}

Coronary angiography: Routine coronary angiography was applied to test the coronary stenosis. Coronary atherosclerotic heart disease was diagnosed by coronary artery diameter $\geq$ $50 \%$ stenosis, including left main branch, anterior descending branch, left circumflex artery and branch, and right coronary artery and branch. The position and degree stenosis, and the number of damaged coronary artery vessels were recorded.

Serum Hcy level detection: The peripheral venous blood was collected in the EDTA-Na2 anticoagulant tube. After centrifuged at $4000 \mathrm{rpm} / \mathrm{min}$ for $15 \mathrm{~min}$, the upper serum was collected for measuring serum Hcy level by an ELISA kit (Ningbo Ruiyuan Biotechnology Co., Ltd, Ningbo, China) according to manufacturer's instructions on an automatic biochemical analyzer (Beckman, model AU480, USA). The sensitivity was $1 \mu \mathrm{mol} / \mathrm{L}$ with accuracy relative deviation of $\leq$ \pm 15\%. Hyperhomocysteinemia (HHcy) graduation: mild, 16-30 $\mu \mathrm{mol} / \mathrm{L}$; moderate, 30-100 $\mu \mathrm{mol} / \mathrm{L}$; severe, $>100$ $\mu \mathrm{mol} / \mathrm{L}$.

\section{Blood lipid level detection}

The peripheral venous blood was collected in the EDTA-Na2 anticoagulant tube. After centrifuged at $4000 \mathrm{rpm} / \mathrm{min}$ for 15 min, the upper serum was collected and analysed on biochemical analyzer according to the manual (HI7100, Japan), including TP, TG, HDL, and LDL. The intra-precision CV was $\leq 5 \%$, inter relative deviation was $\leq 7 \%$ and relative deviation of accuracy was $\leq \pm 15 \%$.

\section{Data analysis}

All data analyses were performed on SPSS 17.0 statistical software. The enumeration data were compared by $\chi^{2}$ test, the measurement data were tested by variance analysis, and the correlation was performed using Pearson correlation test. $\mathrm{P}<0.05$ was considered as statistical significance.

\section{Results}

\section{Serum Hcy and blood lipid levels in patients with different clinical types of $\mathrm{CHD}$}

It was found that Hcy, TP, TG, and LDL were significantly higher, while HDL level was obviously lower in experimental group compared with control $(\mathrm{P}<0.05)$. The levels of serum Hcy, TP, TG, and LDL gradually increased, whereas HDL level gradually declined among SAP, UAP, and AMI patients $(\mathrm{P}<0.05)$ (Figure 1).

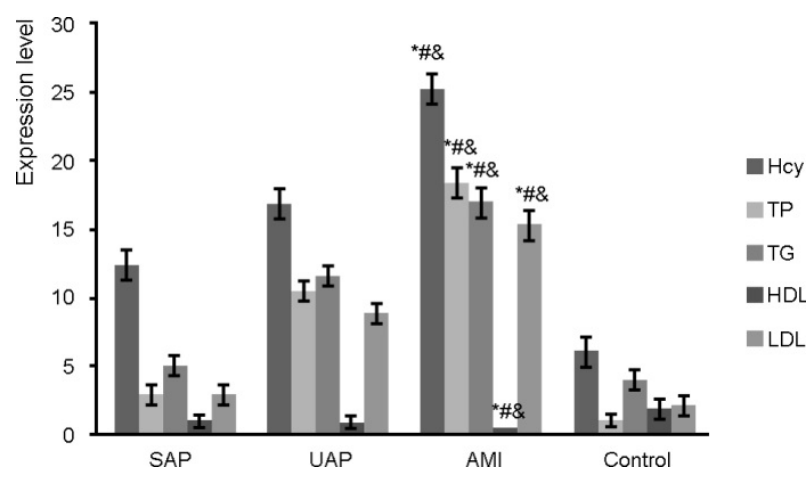

Figure 1. Serum Hcy and blood lipid levels in patients with different clinical types of CHD. ${ }^{*} P<0.05$, compared with control; ${ }^{\#} P<0.05$, compared with SAP group; ${ }^{\&} P<0.05$, compared with UAP group.

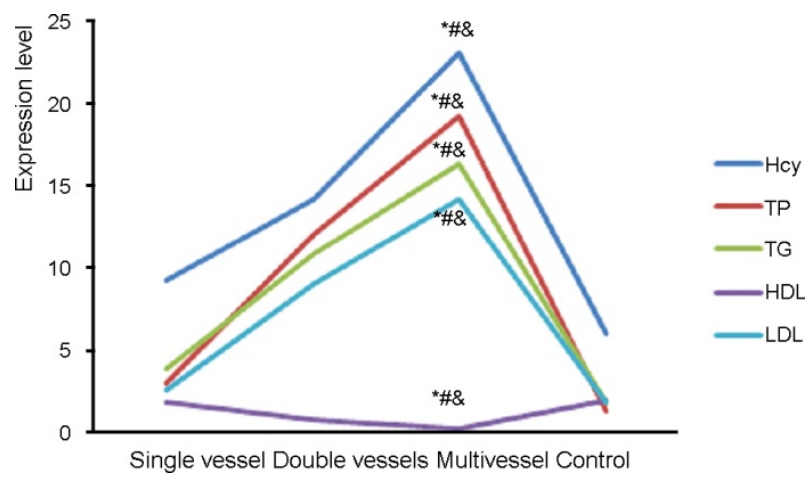

Figure 2. Serum Hcy and blood lipid levels in CHD patients with different numbers of vascular lesions. ${ }^{*} P<0.05$, compared with control; ${ }^{\#} P<0.05$, compared with single vessel group; ${ }^{\&} P<0.05$, compared with double vessels group.

\section{Serum Hcy and blood lipid levels in CHD patients with different numbers of vascular lesions}

It was showed that the levels of Hcy, TP, TG, HDL, and LDL were markedly higher in the patients with different number of vascular lesions than those in the control group $(\mathrm{P}<0.05)$. The levels of Hcy, TP, TG, and LDL gradually upregulated, while 
Diagnostic value of serum homocysteine and blood lipid level in different types of coronary atherosclerotic cardiopathy

HDL level gradually decreased in single vessel disease group, double vessels disease group, and multivessel disease group $(\mathrm{P}<0.05)$ (Figure 2).

Table 1. Serum Hcy and blood lipid levels in CHD patients with different coronary stenosis.

\begin{tabular}{|c|c|c|c|c|c|c|}
\hline Group & Cases & Hcy ( $\mu \mathrm{mol} / \mathrm{l})$ & TP (mmol/l) & TG (mmol/l) & HDL (mmol/l) & LDL (mmol/l) \\
\hline Experimental group & 100 & & & & & \\
\hline Mild stenosis & 21 & $6.33 \pm 0.84^{*}$ & $2.85 \pm 1.02^{*}$ & $2.93 \pm 1.01^{*}$ & $1.22 \pm 0.76^{*}$ & $2.89 \pm 1.13^{*}$ \\
\hline Moderate stenosis & 29 & $10.45 \pm 1.16^{* \#}$ & $8.78 \pm 1.67^{* \#}$ & $9.56 \pm 1.17^{* \#}$ & $0.83 \pm 0.43^{* \#}$ & $8.37 \pm 1.31^{* \#}$ \\
\hline Severe stenosis & 32 & $14.79 \pm 3.22^{* \# \&}$ & $11.25 \pm 3.48^{* \# \&}$ & $12.45 \pm 3.02^{* \# \&}$ & $0.37 \pm 0.04^{* \# \&}$ & $13.46 \pm 3.02^{* \# \&}$ \\
\hline Occlusion & 18 & $17.03 \pm 5.01^{*} \# \& @$ & $15.24 \pm 3.88^{* \# \& @ ~}$ & $17.03 \pm 4.89^{*} \# \& @$ & $0.12 \pm 0.01^{* \# \& @ ~}$ & 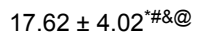 \\
\hline Control & 100 & $1.92 \pm 0.17$ & $1.29 \pm 0.34$ & $1.45 \pm 0.21$ & $1.98 \pm 1.33$ & $1.73 \pm 0.11$ \\
\hline
\end{tabular}

${ }^{*} \mathrm{P}<0.05$, compared with control; ${ }^{*} \mathrm{P}<0.05$, compared with mild stenosis group; ${ }^{\mathrm{P}}<0.05$, compared with moderate stenosis group; @ $\mathrm{P}<0.05$, compared with severe stenosis group.

\section{Serum Hcy and blood lipid levels in CHD patients with different coronary stenosis}

It was demonstrated that serum Hcy and blood lipid levels in patients with different coronary stenosis were higher than those in the control group $(\mathrm{P}<0.05)$. The levels of serum Hcy, TP, TG, and LDL gradually elevated, while HDL level gradually reduced in patients with mild, moderate, severe stenosis, and entirely occlusion $(\mathrm{P}<0.05)$ (Table 1$)$.

\section{Sensitivity and specificity of serum Hcy and blood lipid levels comparison in CHD patients}

It was exhibited that the sensitivity of HDL in CHD patients was obviously higher than that of serum Hcy, TP, TG, and LDL. On the other side, the specificity of serum Hcy was markedly higher than that of TP, TG, HDL, and LDL. TP, TG, and LDL showed relative low sensitivity and specificity (Table 2).

\section{The correlation relationship between serum Hcy and blood lipid levels in CHD patients}

Serum Hcy demonstrated no statistical correlation with blood lipid level ( $\mathrm{P}>0.05)$ (Table 3$)$.

Table 2. Sensitivity and specificity of serum Hcy and blood lipid levels comparison in CHD patients.

\begin{tabular}{lll}
\hline Item & Sensitivity (\%) & Specificity (\%) \\
\hline Hcy & 73.5 & 91.5 \\
\hline TP & 67.5 & 59.1 \\
\hline TG & 34.5 & 86.3 \\
\hline HDL & 91.4 & 69.2 \\
\hline LDL & 31.1 & 81.3 \\
\hline
\end{tabular}

Table 3. The correlation relationship between serum Hcy and blood lipid levels in CHD patients.

\begin{tabular}{lll}
\hline \multirow{2}{*}{ Item } & Hcy & \\
\cline { 2 - 3 } & $\mathbf{r}$ & $\mathbf{P}$ \\
\hline TP & 0.148 & 0.391 \\
\hline TG & 0.167 & 0.336 \\
\hline HDL & 0.148 & 0.398 \\
\hline LDL & 0.029 & 0.863 \\
\hline
\end{tabular}

\section{Discussion}

In recent years, coagulation system and fibrinolytic system abnormalities, vascular endothelial cells damage, and acute and chronic inflammatory and infectious diseases are involved in the entire pathophysiology process of CHD [8,9]. Currently, coronary angiography is the gold standard for the diagnosis of CHD. However, its application in clinic is limited by price and invasiveness. Hcy is the product of methionine but does not participate in proteins synthesis [10]. It was showed that Hcy level is an independent risk factor for the development of cardiovascular disease. Hcy upregulation in the blood can stimulate the blood vessel wall, resulting in arterial damage, and ultimately leading to the production of inflammatory diseases and intravascular plaque [11]. In this study, we selected CHD patients and examined the relationship between serum Hcy and blood lipid levels in the development of different types of CHD by testing Hcy and blood lipid levels.

In this study, we selected CHD patients as experimental group and healthy volunteers as normal control to compare serum Hcy, TP, TG, HDL, and LDL levels in different clinical types, numbers of vascular lesions, and degree of coronary stenosis. It was found that Hcy, TP, TG, and LDL were significantly higher, while HDL level was obviously lower in experimental group compared with control. The levels of serum Hcy, TP, TG, and LDL gradually increased, whereas HDL level gradually declined among SAP, UAP, and AMI patients. The levels of Hcy, TP, TG, and LDL gradually upregulated, while HDL level gradually decreased in single vessel disease group, 
double vessels disease group, and multivessel disease group. The levels of serum Hcy, TP, TG, and LDL gradually elevated, while HDL level gradually reduced in patients with mild, moderate, severe stenosis, and entirely occlusion. It suggested that Hcy level increased in CHD patients, accompanied by blood lipid elevation, especially in patients with AMI, multivessel disease, and even entirely occlusion. Lipid index is a direct reflection of abnormal lipid metabolism in CHD patients. Hyperlipidemia may increase LDL synthesis, accompanied by coagulation factor synthesis and activation, hypercoagulable state, and the changes in hemorheology, which further aggravates the incidence and development of CHD $[12,13]$. Several studies showed that Hcy is a key factor in the development of CHD. It was confirmed that Hcy plays a role in damaging vascular wall, affecting coagulation factors, and inducing lipid accumulation. High level of Hcy is a risk factor for the development of atherosclerotic disease [14,15]. It was showed that in the pathogenesis of CHD, Hcy can produce oxidation, release hydrogen peroxide and superoxide anion, inhibit nitric oxide synthesis, and promote nitric oxide degradation, leading to abnormal vascular function; secondly, LDL-C formed a combination with Hcy product, which came into atherosclerotic plaque through aggregation; thirdly, Hcy reduced the function of the vascular endothelium and increased its procoagulant effect, resulting in the imbalance of the coagulation and fibrillation system [16-18].

This study further compared the sensitivity and specificity of serum Hcy and blood lipid levels in CHD patients. The sensitivity of HDL in CHD patients was obviously higher than that of serum Hcy, TP, TG, and LDL. On the other side, the specificity of serum Hcy was markedly higher than that of TP, TG, HDL, and LDL. TP, TG, and LDL showed relative low sensitivity and specificity. At last, this research analysed the correlation between serum Hcy and blood lipid levels, and observed no statistical correlation between them. It indicated that serum Hcy and HDL could be treated as crucial reference in the diagnosis of CHD. Though no significant correlation was found between Hcy and blood lipid level, multiple studies revealed that the onset risk of CHD increased following the elevation of serum Hcy and blood lipid. The development of $\mathrm{CHD}$ is a chronic complex pathophysiological process, which is the result of multi-factors participation and interaction. The occurrence and development of CHD is related to various aspects, including lipid metabolism, coagulation and fibrillation imbalance, endothelial cell injury, inflammation, and infection $[16,19,20]$. We found that high levels of Hcy expression and blood lipids exhibited diagnostic value on different types of CHD. Combined detection, including serum Hcy and blood lipid-related laboratory indicators, can improve the accuracy of CHD diagnosis, which is helpful to more correctly evaluate of the occurrence, development, and prognosis of CHD.

\section{Conclusion}

Hcy level increased in CHD patients, accompanied by blood lipid elevation, especially in patients with AMI, multivessel disease, and even entirely occlusion. Serum HDL exhibited high sensitivity, while serum Hcy presented high specificity. Serum Hcy demonstrated no statistical correlation with blood lipid level. In-depth investigation of the occurrence and development of $\mathrm{CHD}$ provides more scientific basis to promoting the faster recovery of CHD patients. In addition, due to limited number of patients enrolled, large cohort study is required to confirm these findings.

\section{References}

1. Idriss NK, Blann AD, Lip GY. Hemoxygenase-1 in cardiovascular disease. J Am Coll Cardiol 2008; 52: 971-978.

2. Sanchez LD, McGillicuddy DC, Volz KA, Fan SL, Joyce N, Horowitz GL. Effect of two different FDA-approved Ddimer assays on resource utilization in the emergency department. Acad Emerg Med 2011; 18: 317-321.

3. Li X, Zhang X, Wei L, Xia Y, Guo X. Relationship between serum oncostatin $\mathrm{M}$ levels and degree of coronary stenosis in patients with coronary artery disease. Clin Lab 2014; 60: 113-118.

4. Sen U, Mishra PK, Tyagi N, Tyagi SC. Homocysteine to hydrogen sulfide or hypertension. Cell Biochem Biophys 2010; 57: 49-58.

5. Porapakkham P, Porapakkham P, Zimmet H, Billah B, Krum H. B-type natriuretic peptide-guided heart failure therapy: a meta-analysis. Arch Intern Med 2010; 170: 507-514.

6. O'Malley RG, Bonaca MP, Scirica BM, Murphy SA, Jarolim P, Sabatine MS, Braunwald E, Morrow DA. Prognostic performance of multiple biomarkers in patients with non-ST-segment elevation acute coronary syndrome: analysis from the MERLIN-TIMI 36 trial (Metabolic efficiency with ranolazine for less ischemia in non-stelevation acute coronary syndromes-thrombolysis in myocardial infarction 36). J Am Coll Cardiol 2014; 63: 1644-1653.

7. Vrentzos G, Papadakis JA, Malliaraki N, Zacharis EA, Katsogridakis K, Margioris AN, Vardas PE, Ganotakis ES. Association of serum total homocysteine with the extent of ischemic heart disease in a Mediterranean cohort. Angiology 2004; 55: 517-524.

8. Eikelboom JW, Lonn E, Genest J, Hankey G, Yusuf S. Homocyst(e)ine and cardiovascular disease: a critical review of the epidemiologic evidence. Ann Intern Med 1999; 131: 363-375.

9. Graham I. Homocysteine in health and disease. Ann Intern Med 1999; 131: 387-388.

10. Terzic J, Grivennikov S, Karin E, Karin M. Inflammation and colon cancer. Gastroenterology 2010; 138: 2101-2114.

11. Zheng JL, Lu L, Hu J, Zhang RY, Zhang Q, Chen QJ, Shen WF. Increased serum YKL-40 and C-reactive protein levels are associated with angiographic lesion progression in patients with coronary artery disease. Atherosclerosis 2010; 210: 590-595. 


\section{cardiopathy}

12. Hartmann M, von Birgelen C, Mintz GS, Stoel MG, Eggebrecht H, Wieneke H, Fahy M, Neumann T, van der Palen J, Louwerenburg HW, Verhorst PM, Erbel R. Relation between lipoprotein(a) and fibrinogen and serial intravascular ultrasound plaque progression in left main coronary arteries. J Am Coll Cardiol 2006; 48: 446-452.

13. Carey VJ, Bishop L, Laranjo N, Harshfield BJ, Kwiat C, Sacks FM. Contribution of high plasma triglycerides and low high-density lipoprotein cholesterol to residual risk of coronary heart disease after establishment of low-density lipoprotein cholesterol control. Am J Cardiol 2010; 106: 757-763.

14. Stankovic S, Majkic-Singh N. Genetic aspects of ischemic stroke: coagulation, homocysteine, and lipoprotein metabolism as potential risk factors. Crit Rev Clin Lab Sci 2010; 47: 72-123.

15. Aroni K, Ioannidis E, Voudouris S, Yapijakis C. Homocysteinemia-associated anetoderma, in a young woman with anorexia nervosa history. Int J Dermatol 2011; 50: 343-345.

16. Garcia-Alonso FJ, Jorge-Vidal V, Ros G, Periago MJ. Effect of consumption of tomato juice enriched with n-3 polyunsaturated fatty acids on the lipid profile, antioxidant biomarker status, and cardiovascular disease risk in healthy women. Eur J Nutr 2012; 51: 415-424.

17. He M, van Dam RM, Rimm E, Hu FB, Qi L. Whole-grain, cereal fiber, bran, and germ intake and the risks of all-cause and cardiovascular disease-specific mortality among women with type 2 diabetes mellitus. Circulation 2010; 121: 2162-2168.

18. Mansikkaniemi K, Juonala M, Taimela S, Hirvensalo M, Telama R, Huupponen R, Saarikoski L, Hurme M, Mallat Z, Benessiano J, Jula A, Taittonen L, Marniemi J, Kahonen M, Lehtimaki T, Ronnemaa T, Viikari J, Raitakari OT. Cross-sectional associations between physical activity and selected coronary heart disease risk factors in young adults. The cardiovascular risk in Young Finns study. Ann Med 2012; 44: 733-744.

19. Greenwood JP, Maredia N, Younger JF, Brown JM, Nixon J, Everett CC, Bijsterveld P, Ridgway JP, Radjenovic A, Dickinson CJ, Ball SG, Plein S. Cardiovascular magnetic resonance and single-photon emission computed tomography for diagnosis of coronary heart disease (CEMARC): a prospective trial. Lancet 2012; 379: 453-460.

20. Jacob T, Hingorani A, Ascher E. Evidence for telomerase activation in VSMCs exposed to hyperglycemic and hyperhomocysteinemic conditions. Angiology 2009; 60: 562-568.

\section{*Correspondence to}

Jun Yang

Qingdao University

Qingdao

PR China 